\title{
DEPOSITIONAL ENVIRONMENT OF THE GOMBE FORMATION IN THE GONGOLA SUB-BASIN OF THE NORTHERN BENUE TROUGH: USING GRAIN SIZE PARAMETERS
}

\author{
M. B. USMAN, Y. D. MAMMAN, U. ABUBAKAR, A. SULAIMAN AND H. HAMIDU
}

(Received 16 June 2016; Revision Accepted 25 July 2016)

\begin{abstract}
The depositional environment of the Gombe Formation was determined using grain size parameters in which sixteen sandstone samples and ninety nine pebbles were subjected to granulometric and pebbles morphometric analysis respectively. The granulometric analysis for the sixteen (16) samples of the Gombe Formation show an average graphic mean of $2.51 \phi$ (fine grained sandstone), mean standard deviation of $0.58 \phi$ (moderately well sorted sandstone), mean skewness value of $0.09 \phi$ (nearly symmetrical) and mean kurtosis value of $0.89 \phi$ (platykurtic). The Bivariate plot of standard deviation vs. skewness indicated dominance of fluvial environment. While the probability curves plots showed a dominance of three sand populations indicating influence of marine processes. Environmental discrimination formulae for $\mathrm{Y} 1, \mathrm{Y} 2$ and $\mathrm{Y} 3$ indicated dominance of Aeolian, shallow agitated marine environment and shallow marine environment respectively. The plots of $Y 2$ vs.Y1 and Y3 vs. Y2 showed a dominance shallow marine environment. The morphometric analysis indicates both fluvial and beach environment with dominance of fluvial environment.
\end{abstract}

KEYWORDS: Gombe Formation, Gongola Sub-Basin, Pebbles Morphology, Granulometric analysis, grain size

\section{INTRODUCTION}

The Benue Trough trends NE - SW for about $1000 \mathrm{~km}$ in length and $150 \mathrm{~km}$ width. It formed during early Cretaceous rifting and strike slip movement of central West African Basement (Benkhelil, 1989). Benue Trough is bounded by Niger-Delta Basin at the southern end and by Chad Basin to the North (Fig. 1) (Zaborski 1998). The trough is a sedimentary basin containing up to $6000 \mathrm{~m}$ of Cretaceous-Tertiary sediments associated with volcanic. The Benue Trough of Nigeria is part of the West and Central African Rift System (WCARS); formed during separation of the African and South American plates during the Early Cretaceous (Fitton, 1983; Genik, 1992).

The Benue Trough is divided into Lower, Middle, and Upper (Fig.1) by Zaborski (1998) while Nwajide (2013) subdivided it into Southern, Central and Northern (this subdivision was used in this study). The Northern Benue Trough is made up of two major sub-basins: the N-S trending Gongola Sub-basin and E-W trending Yola sub-basin (Nwajide, 2013). The Geology and stratigraphy of Northern Benue Trough was described in detail by Carter el al. (1963), Offodile (1976), Benkhelil (1989), Zaborski et al. (1997), Abubakar (2006) and
Tukur et al. (2015). The stratigraphic succession in the Gongola sub-basin of the Northern Benue Trough comprises the Aptian-Albian Continental Bima Formation, The Cenomanian transitional-marine Yolde Formation, the Cenomanian-Santonian marine Pindiga Formation, the Campano-Maastrichtian Deltaic Gombe Formation (the formation of interest in this study) and Tertiary Continental Keri-Keri Formation (Fig. 2).

In the study area the environment of deposition of Gombe Formation was interpreted as fluvial dominated delta by Carter el al. (1963) and Abubakar (2006). Shetima et al. (2012) regarded the environment of deposition of the formation as marginal marine based on textural and lithologic analysis. Hamidu (2012) regarded red sandstone facies, bedded facies and interbedded facies of Zaborski et al. (1997) as alluvial, beach, shoreface, and sublitoral environment respectively.

The study area is located mainly at Gombe and Environs in the Gongola sub-basin. The purpose of this study is to use pebble morphometric analysis and Sahu (1964) method in addition to granulometric analysis used by Shetima et al. (2012) to interpret the depositional environment of Campano-Maastrichtian Gombe Formation.

\footnotetext{
M. B. Usman, Department of Geology, Gombe State University, Gombe, Nigeria

Y. D. Mamman, Department of Geology, Modibbo Adama University of Technology, Yola, Nigeria

U. Abubakar, Department of Geology, Gombe State University, Gombe, Nigeria

A. Sulaiman, Department of Geology, Gombe State University, Gombe, Nigeria

H. Hamidu, Department of Geology, Usmanu Danfodiyo University Sokoto, Nigeria
} 


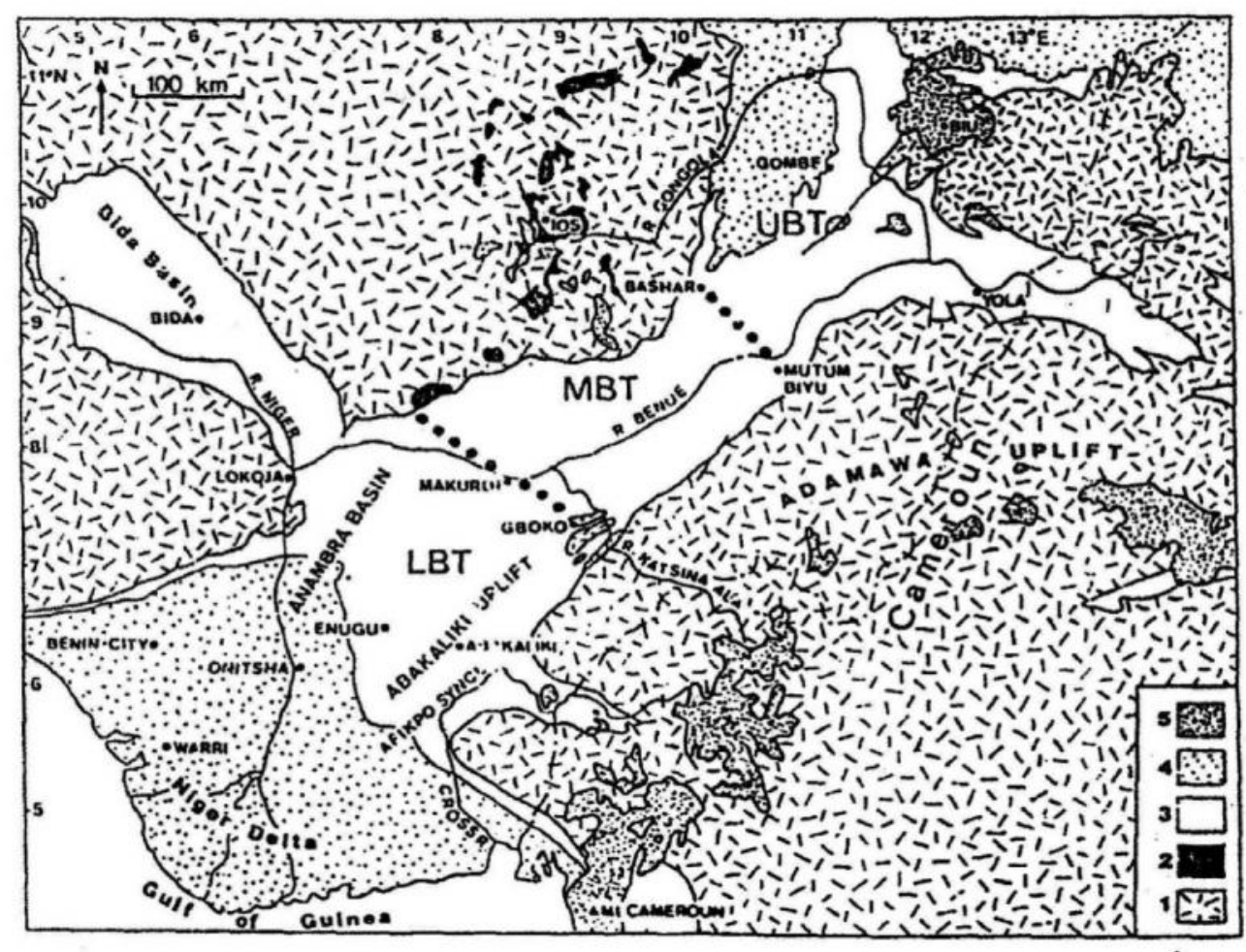

Figure 1: Outline of Geological Map of the Benue Trough and adjacent areas. LBT Lower Benue Trough; MBT Middle Benue Trough; UBT Upper Benue Trough. 1. Precambrian. 2. Jurassic "Younger Granites" 3. Cretaceous 4. Post Cretaceous sediments 5. Cenozoic Recent basalts including those of Cameroon Line (after Zaborski, 1998).

\begin{tabular}{|c|c|c|c|c|c|}
\hline Age & $\begin{array}{c}\text { Formation } \\
\text { (Gongola Arm) }\end{array}$ & $\begin{array}{l}\text { Formation } \\
\text { (Yola Arm) }\end{array}$ & Lithology & \multicolumn{2}{|c|}{ Paleoenvironment } \\
\hline $\begin{array}{l}\text { Tertiary } \\
\ldots . . . . . . . . .\end{array}$ & \multirow{3}{*}{$\begin{array}{l}\text { Kerri Kerri } \\
\text { Gombe } \\
\text { Sandstone }\end{array}$} & \multirow{3}{*}{ Erosion? } & & \multicolumn{2}{|c|}{$\begin{array}{c}\text { Continental } \\
\text { (Fluvial/Lacustrinel }\end{array}$} \\
\hline Maastrictian & & & & \multirow{2}{*}{\multicolumn{2}{|c|}{$\begin{array}{c}\text { Continental } \\
\text { (Lacustrine/Deltaic) }\end{array}$}} \\
\hline Companian & & & menses & & \\
\hline Santonain & \multirow{3}{*}{ 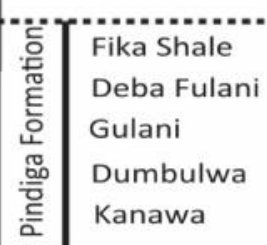 } & \multirow{3}{*}{$\begin{array}{l}\text { Lamja } \\
\text { Numanha } \\
\text { Sekuliye } \\
\text { Jessu } \\
\text { Dukkul }\end{array}$} & $\infty$ & \multirow{3}{*}{\multicolumn{2}{|c|}{$\begin{array}{c}\text { Marine } \\
\text { (Offshore/Estuarine }\end{array}$}} \\
\hline Coniacian & & & & & \\
\hline Turinian & & & & & \\
\hline Cenomanian & \multicolumn{2}{|l|}{ Yolde } & & \multicolumn{2}{|c|}{ Transitional } \\
\hline \multirow{2}{*}{$\begin{array}{l}\text { Albian and } \\
\text { older }\end{array}$} & \multicolumn{2}{|c|}{ Upper Bima Sandstone Member } & 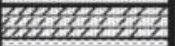 & Braided & \\
\hline & \multicolumn{2}{|c|}{ Lower Bima Sandstone Member } &  & $\begin{array}{l}\text { Alluvial/Braided/ } \\
\text { Lacustrine }\end{array}$ & Continental \\
\hline Precambrian & \multicolumn{2}{|c|}{ Basement Complex } & & \multicolumn{2}{|c|}{ Igneous/Metamorphic } \\
\hline
\end{tabular}

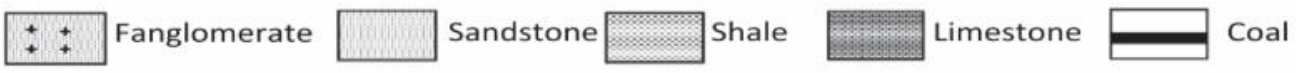

$>>$ Ferruginized sandstone $\quad \begin{aligned} & ++ \\ & +++\end{aligned}$

Unconformity

Figure 2: Stratigraphy succession of Benue Trough (Tukur et al. 2015) 


\section{GEOLOGICAL SETTING}

The Gombe Formation is the youngest Cretaceous lithostratigraphic unit in the N-S trending Gongola sub-basin of the Northern Benue Trough (Popoff et al. 1986 and Nwajide 2013). It unconformably overlies Fika Shale and is overlain by Palaeocene KeriKeri Formation. The lithologic units of the Gombe Formation are divided into three (Zaborski et al. 1997): basal interbedded unit, middle bedded facie and upper red sandstone facies. The basal unit comprise alternating thin beds of silty shalves, with some plant remains and fine to medium grained sandstone intercalated with flaggy ironstones. The middle part consists of regularly horizontally bedded, fine to medium grained quartz arenite, interbedded with Silts, Silty Clays and ironstones while, the upper part of the Formation consists of brick red coloured sandstone. The grain sizes range from pebble to medium grained sandstones with trough, tabular, and planar cross-bedding. The Gombe Formation is regarded as Maastrichtian in age (Carter et al. 1963; Kogbe 1976; Popoff et al. 1986).

\section{METHODS}

Sixteen (16) sandstones samples and ninety nine pebbles were collected from four outcrop sections of Gombe Formation for granulometric analysis and pebble morphometric study respectively. Samples obtained from Arawa stream, Pantami stream and Mallam Sidi road cut setion were sieved using Folk and Ward (1957) method. The graphical parameters of graphic mean, standard deviation, skewness, and kurtosis were calculated using Folk and Ward (1957) formula. The calculated graphical parameters were used for palaeoenvironment discrimination using the method proposal by Sahu (1964). The following Sahu (1964) formulae were used for palaeoenvironmental discrimination;

$$
\begin{aligned}
\mathrm{Y}_{1}= & -3.5688 \mathrm{MZ}+3.7016 \delta_{1}{ }^{2}-2.0766 \mathrm{SK}_{1}+ \\
& 3.1135 \mathrm{KG} \\
\mathrm{Y}_{2}= & 15.6534 \mathrm{MZ}+65.7091 \delta_{1}{ }^{2}+18.1071 \mathrm{SK}_{1}+ \\
& 18.5043 \mathrm{KG} \\
\mathrm{Y}_{3}= & \begin{array}{l}
0.2852 \mathrm{MZ}-8.7604 \delta_{1}{ }^{2}-4.8932 \mathrm{SK}_{1}+ \\
0.0482 \mathrm{KG}
\end{array}
\end{aligned}
$$

Where MZ: mean value, $\delta_{1}{ }^{2}$ : standard deviation, SK1: Skewness and KG: kurtosis
The bivariate plots of Friedman (1979), Sahu (1964) and Visher (1969) sand population were applied to interpret the depositional environments of Gombe Formation.

In pebbles morphometric analysis, the long axis (a), the intermediate axis (b) and the short axis (c) were measured with vernier calliper according to Wentworth (1922) method. The values obtained were used for computing the statistical parameters based on Zingg (1935), Wentworth (1922) and Dobkins and Folks (1970). Axial ration were calculated according to Zingg (1935). Maximum projection sphericity and coefficient of flatness were calculated according to the method of Dobkins and Folks (1970) and Stratten (1974) respectively.

\section{RESULTS}

The granulometric analysis results of percentile value for the sixteen sandstone samples of Gombe Formation are presented in Table 1. The graphic mean, Standard deviation, skewness, kurtosis of the analysed samples are presented in Table 2.

The graphic mean for the various samples ranges from $0.8 \phi$ to $3.1 \phi$ with an average of $2.5 \phi$ (fine grained sandstone) (Table 2) which indicate coarse to very fine grained sandstone. The standard deviation values range from $0.4 \phi-1.0 \phi$ (Table 2) with average of $0.6 \phi$ (moderately well sorted sandstone), which indicate well to poorly sorted. However, moderately sorted to well sorted values predominate. The values for the skewness range from $-0.3 \phi$ to $0.4 \phi$ (Table 2) which indicate negative skewed to positive skewed. However the positive skewed values predominate. The values of Kurtosis for the various sample range from $0.6 \phi$ to $2.1 \phi$ (very platykurtic to very leptokurtic) with an average of $1.3 \phi$ (leptokurtic). The Bivariate plot of standard deviation vs. skewness indicated dominance of fluvial environment (fig.3). While the probability curves plots showed a dominance of three sand populations and subordinate results of two sand populations (figs. 4). The results obtained from environmental discrimination functions (Y1, Y2 and Y3) of Sahu (1964) are presented in Table 3. The values of maximum projection sphericity and coefficient of flatness obtained are shown in Table 4,5 and 6. 
Table 1: Percentile value from cumulative curve plots of sandstone samples analyzed.

\begin{tabular}{llllllll}
\hline Samples & $\phi 5$ & $\phi 16$ & $\phi 25$ & $\phi 50$ & $\phi 75$ & $\phi 84$ & $\phi 95$ \\
\hline 1 & 1.4 & 2.7 & 2.8 & 3.1 & 3.3 & 3.5 & 3.8 \\
2 & 2.0 & 2.2 & 2.3 & 2.6 & 3.1 & 3.3 & 4.3 \\
3 & 1.0 & 2.0 & 2.2 & 2.4 & 2.7 & 3.0 & 3.5 \\
5 & 2.0 & 2.1 & 2.2 & 2.4 & 2.7 & 3.0 & 3.5 \\
6 & 2.0 & 2.1 & 2.2 & 2.4 & 2.6 & 2.8 & 3.3 \\
7 & 2.0 & 2.2 & 2.3 & 2.6 & 3.1 & 3.3 & 3.6 \\
9 & 2.0 & 2.2 & 2.2 & 2.5 & 2.9 & 3.1 & 3.5 \\
10 & 2.1 & 2.3 & 2.5 & 2.9 & 3.3 & 3.4 & 3.7 \\
11 & 2.0 & 2.3 & 2.5 & 3.0 & 3.4 & 3.5 & 3.9 \\
12 & 1.0 & 2.7 & 2.8 & 3.1 & 3.3 & 3.4 & 3.8 \\
13 & 2.0 & 2.2 & 2.3 & 2.6 & 3.0 & 3.2 & 3.4 \\
14 & 1.0 & 2.8 & 2.8 & 2.9 & 3.3 & 3.5 & 3.8 \\
15 & 2.1 & 2.3 & 2.5 & 2.9 & 3.2 & 3.3 & 3.5 \\
16 & 1.2 & 1.3 & 1.9 & 2.3 & 2.7 & 3.0 & 3.6 \\
17 & 0.5 & 0.0 & 0.3 & 0.8 & 1.4 & 1.6 & 2.0 \\
18 & 0.0 & 0.0 & 0.7 & 1.3 & 1.9 & 2.2 & 3.0 \\
\hline
\end{tabular}


Table 2 Grain size distribution and quantitative parameters for the sandstone samples analyzed

\begin{tabular}{|c|c|c|c|c|}
\hline Samples Id & Mean & $\begin{array}{l}\text { Standard } \\
\text { Deviation } \\
\text { (Sorting) }\end{array}$ & Skewness (SK1) & Kurtosis (KG) \\
\hline 1 & $\begin{array}{l}3.10 \\
\text { Very fine sand }\end{array}$ & $\begin{array}{l}0.54 \\
\text { Moderately well } \\
\text { sorted sand }\end{array}$ & $\begin{array}{l}-0.21 \\
\text { Positive skewed }\end{array}$ & $\begin{array}{l}2.05 \\
\text { Very leptokurtic }\end{array}$ \\
\hline 2 & $\begin{array}{l}2.70 \\
\text { Fine sand }\end{array}$ & $\begin{array}{l}0.64 \\
\text { Moderately well } \\
\text { sorted sand }\end{array}$ & $\begin{array}{l}0.35 \\
\text { Strong } \quad \text { Positive } \\
\text { skewed }\end{array}$ & $\begin{array}{l}1.11 \\
\text { Mesokurtic }\end{array}$ \\
\hline 3 & $\begin{array}{l}2.50 \\
\text { Fine sand }\end{array}$ & $\begin{array}{l}0.61 \\
\text { Moderately well } \\
\text { sorted sand }\end{array}$ & $\begin{array}{l}0.09 \\
\text { Near symmetrical }\end{array}$ & $\begin{array}{l}1.86 \\
\text { Very leptokurtic }\end{array}$ \\
\hline 5 & $\begin{array}{l}2.50 \\
\text { Fine sand }\end{array}$ & $\begin{array}{l}0.45 \\
\text { Well sorted }\end{array}$ & $\begin{array}{l}0.40 \\
\text { Strong } \\
\text { skewed }\end{array}$ & $\begin{array}{l}1.23 \\
\text { Leptokurtic }\end{array}$ \\
\hline 6 & $\begin{array}{l}2.43 \\
\text { Fine sand }\end{array}$ & $\begin{array}{l}0.37 \\
\text { Well sorted }\end{array}$ & $\begin{array}{l}0.26 \\
\text { Positive skewed }\end{array}$ & $\begin{array}{l}1.33 \\
\text { Leptokurtic }\end{array}$ \\
\hline 7 & $\begin{array}{l}2.69 \\
\text { Fine sand }\end{array}$ & $\begin{array}{l}0.52 \\
\text { Moderately well } \\
\text { sorted sand }\end{array}$ & $\begin{array}{l}0.25 \\
\text { Positive skewed }\end{array}$ & $\begin{array}{l}0.82 \\
\text { Platykurtic }\end{array}$ \\
\hline 9 & $\begin{array}{l}2.58 \\
\text { Fine sand }\end{array}$ & $\begin{array}{l}0.47 \\
\text { Well sorted }\end{array}$ & $\begin{array}{l}0.30 \\
\text { Positive skewed }\end{array}$ & $\begin{array}{l}0.90 \\
\text { Platykurtic }\end{array}$ \\
\hline 10 & $\begin{array}{l}2.87 \\
\text { Fine sand }\end{array}$ & $\begin{array}{l}0.52 \\
\text { Moderately well } \\
\text { sorted sand }\end{array}$ & $\begin{array}{l}-0.05 \\
\text { Near Symmetrical }\end{array}$ & $\begin{array}{l}0.86 \\
\text { Platykurtic }\end{array}$ \\
\hline 11 & $\begin{array}{l}2.93 \\
\text { Fine sand }\end{array}$ & $\begin{array}{l}0.59 \\
\text { Moderately well } \\
\text { sorted sand }\end{array}$ & $\begin{array}{l}-0.11 \\
\text { Negative skewed }\end{array}$ & $\begin{array}{l}0.87 \\
\text { Platykurtic }\end{array}$ \\
\hline 12 & $\begin{array}{l}3.08 \\
\text { Very fine sand }\end{array}$ & $\begin{array}{l}0.59 \\
\text { Moderately well } \\
\text { sorted sand }\end{array}$ & $\begin{array}{l}-0.31 \\
\text { Strong negative } \\
\text { skewed }\end{array}$ & $\begin{array}{l}2.27 \\
\text { Very leptokurtic }\end{array}$ \\
\hline 13 & $\begin{array}{l}2.67 \\
\text { Fine sand }\end{array}$ & $\begin{array}{l}0.46 \\
\text { Well sorted }\end{array}$ & $\begin{array}{l}0.19 \\
\text { Positive skewed }\end{array}$ & $\begin{array}{l}0.79 \\
\text { Platykurtic }\end{array}$ \\
\hline 14 & $\begin{array}{l}3.03 \\
\text { Very fine sand }\end{array}$ & $\begin{array}{l}0.59 \\
\text { Moderately well } \\
\text { sorted sand }\end{array}$ & $\begin{array}{l}0.26 \\
\text { Positive skewed }\end{array}$ & $\begin{array}{l}2.39 \\
\text { Very leptokurtic }\end{array}$ \\
\hline 15 & $\begin{array}{l}2.85 \\
\text { Fine sand }\end{array}$ & $\begin{array}{l}0.48 \\
\text { Well sorted }\end{array}$ & $\begin{array}{l}-0.15 \\
\text { Negative skewed }\end{array}$ & $\begin{array}{l}0.83 \\
\text { Platykurtic }\end{array}$ \\
\hline 16 & $\begin{array}{l}2.20 \\
\text { Fine sand }\end{array}$ & $\begin{array}{l}0.80 \\
\text { Moderately } \\
\text { sorted sand }\end{array}$ & $\begin{array}{l}-0.06 \\
\text { Negative skewed }\end{array}$ & $\begin{array}{l}1.26 \\
\text { Leptokurtic }\end{array}$ \\
\hline 17 & $\begin{array}{l}0.80 \\
\text { Coarse sand }\end{array}$ & $\begin{array}{l}0.63 \\
\text { Moderately well } \\
\text { sorted sand }\end{array}$ & $\begin{array}{l}0.30 \\
\text { Positive skewed }\end{array}$ & $\begin{array}{l}0.56 \\
\text { Very platykurtic }\end{array}$ \\
\hline 18 & $\begin{array}{l}1.17 \\
\text { Medium sand }\end{array}$ & $\begin{array}{l}1.01 \\
\text { Poorly sorted }\end{array}$ & $\begin{array}{l}-0.02 \\
\text { Negative skewed }\end{array}$ & $\begin{array}{l}1.03 \\
\text { Mesokurtic }\end{array}$ \\
\hline
\end{tabular}




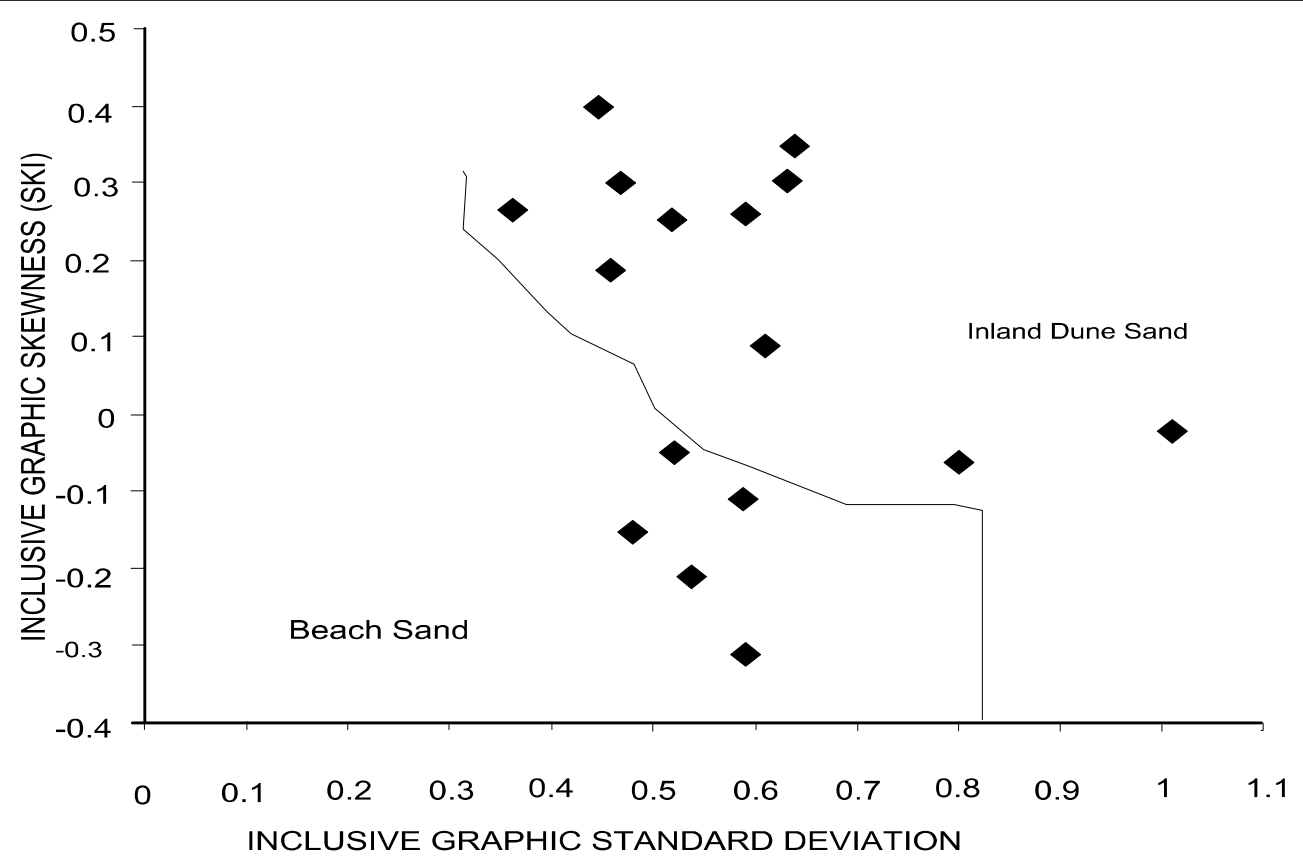

Figure 3: Bivariate plot of skewness vs. standard deviation (after Friedman, 1979)
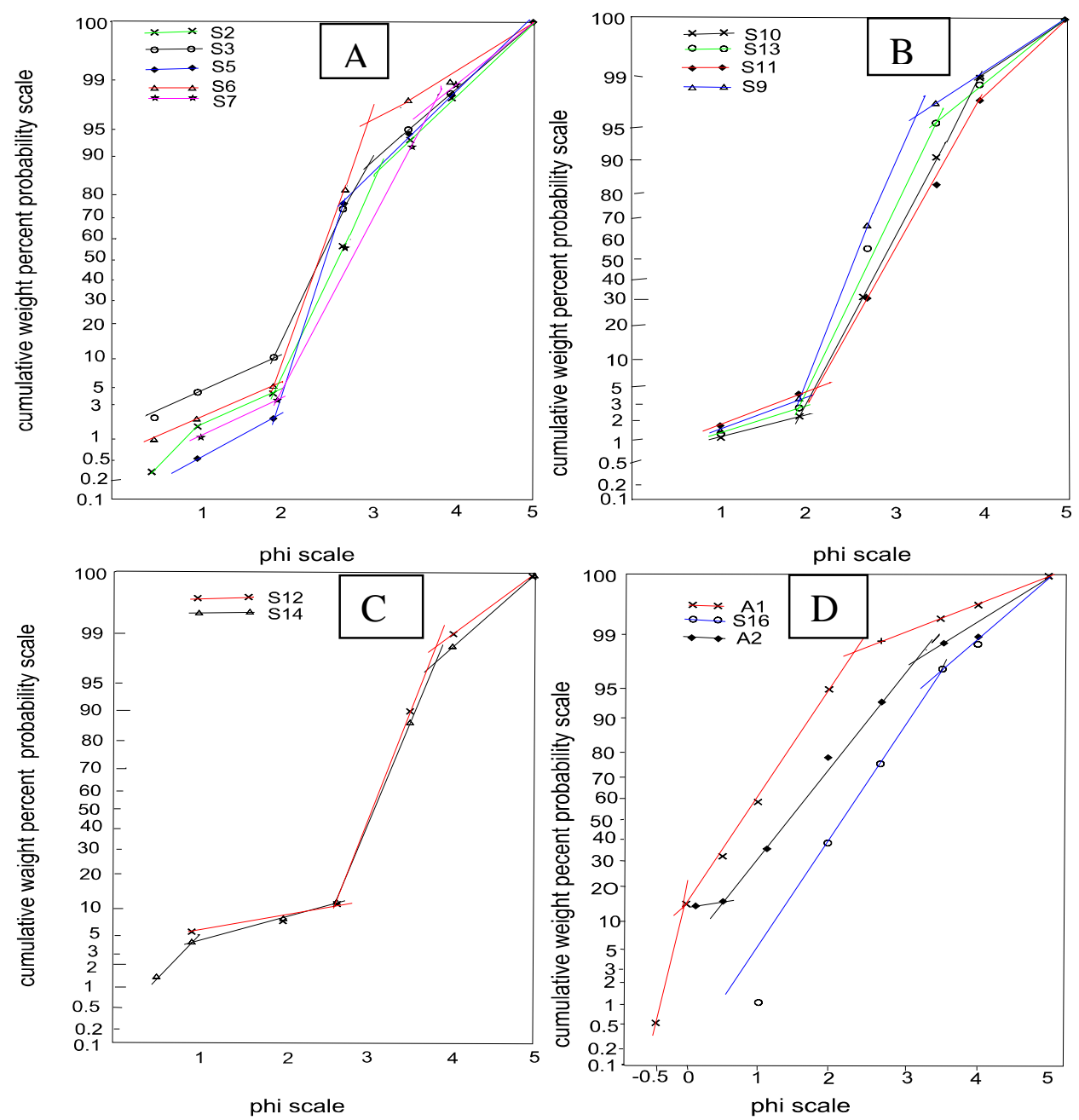

Figure 4: Sand population distribution curves based on log probability plots 
Table 3: The discriminate function and environment of deposition

\begin{tabular}{|c|c|c|c|c|}
\hline Sample no. & $\mathrm{Y} 1$ & & Y2 & Y3 \\
\hline 1 & $\begin{array}{l}-6.75 \\
\text { Aeolian }\end{array}$ & shallow & $\begin{array}{l}80.54 \\
\text { agitated marine }\end{array}$ & $\begin{array}{l}-0.60 \\
\text { shallow marine }\end{array}$ \\
\hline 2 & $\begin{array}{l}-5.32 \\
\text { Aeolian }\end{array}$ & shallow & $\begin{array}{l}95.74 \\
\text { agitated marine }\end{array}$ & $\begin{array}{l}-4.48 \\
\text { shallow marine }\end{array}$ \\
\hline 3 & $\begin{array}{l}-1.94 \\
\text { Beach }\end{array}$ & shallow & $\begin{array}{l}99.63 \\
\text { agitated marine }\end{array}$ & $\begin{array}{l}-2.897 \\
\text { shallow marine }\end{array}$ \\
\hline 5 & $\begin{array}{l}-5.17 \\
\text { Aeolian }\end{array}$ & shallow & $\begin{array}{l}82.44 \\
v \text { agitated marine }\end{array}$ & $\begin{array}{l}-2.96 \\
\text { shallow marine }\end{array}$ \\
\hline \multirow[t]{2}{*}{6} & $\begin{array}{l}-4.57 \\
\text { Aeolian }\end{array}$ & shallow & $\begin{array}{l}76.35 \\
\text { agitated marine }\end{array}$ & $\begin{array}{l}-1.71 \\
\text { shallow marine }\end{array}$ \\
\hline & $\begin{array}{l}-6.57 \\
\text { Aeolian }\end{array}$ & shallow & $\begin{array}{l}79.58 \\
\text { v agitated marine }\end{array}$ & $\begin{array}{l}-2.79 \\
\text { shallow marine }\end{array}$ \\
\hline 9 & $\begin{array}{l}-6.21 \\
\text { Aeolian }\end{array}$ & shallow & $\begin{array}{l}76.99 \\
\text { v agitated marine }\end{array}$ & $\begin{array}{l}-2.62 \\
\text { shallow marine }\end{array}$ \\
\hline 10 & $\begin{array}{l}-6.46 \\
\text { Aeolian }\end{array}$ & shallow & $\begin{array}{l}77.67 \\
\text { v agitated marine }\end{array}$ & $\begin{array}{l}-1.25 \\
\text { shallow marine }\end{array}$ \\
\hline 11 & $\begin{array}{l}-6.23 \\
\text { Aeolian }\end{array}$ & shallow & $\begin{array}{l}82.84 \\
v \text { agitated marine }\end{array}$ & $\begin{array}{l}-1.63 \\
\text { shallow marine }\end{array}$ \\
\hline 12 & $\begin{array}{l}-1.99 \\
\text { Beach }\end{array}$ & shallow & $\begin{array}{l}107.48 \\
v \text { agitated marine }\end{array}$ & $\begin{array}{l}-0.56 \\
\text { shallow marine }\end{array}$ \\
\hline 13 & $\begin{array}{l}-6.68 \\
\text { Aeolian }\end{array}$ & shallow & $\begin{array}{l}73.76 \\
v \text { agitated marine }\end{array}$ & $\begin{array}{l}-1.98 \\
\text { shallow marine }\end{array}$ \\
\hline 14 & $\begin{array}{l}-2.62 \\
\text { Beach }\end{array}$ & shallon & $\begin{array}{l}119.24 \\
\text { w agitated marine }\end{array}$ & $\begin{array}{l}-3.34 \\
\text { shallow marine }\end{array}$ \\
\hline 15 & $\begin{array}{l}-6.42 \\
\text { Aeolian }\end{array}$ & shallow & $\begin{array}{l}72.39 \\
v \text { agitated marine }\end{array}$ & $\begin{array}{l}-0.43 \\
\text { shallow marine }\end{array}$ \\
\hline 16 & $\begin{array}{l}-1.44 \\
\text { Beach }\end{array}$ & shallow & $\begin{array}{l}98.72 \\
\text { agitated marine }\end{array}$ & $\begin{array}{l}-4.63 \\
\text { shallow marine }\end{array}$ \\
\hline 17 & $\begin{array}{l}-0.27 \\
\text { Beach }\end{array}$ & & $\begin{array}{l}54.40 \\
\text { Beach }\end{array}$ & $\begin{array}{l}-4.69 \\
\text { shallow marine }\end{array}$ \\
\hline 18 & $\begin{array}{l}2.86 \\
\text { Beach }\end{array}$ & shallow & $\begin{array}{l}103.97 \\
\text { vaitated marine }\end{array}$ & $\begin{array}{l}-8.44 \\
\text { Fluvial }\end{array}$ \\
\hline
\end{tabular}


Table 4: Summary of pebble morphometric analysis results at $10^{\circ} 24174 \mathrm{~N}$ and $11^{0} 14757 \mathrm{E}$

\begin{tabular}{|c|c|c|c|c|c|c|}
\hline S/No & $\begin{array}{l}\mathrm{a} \\
(\mathrm{cm})\end{array}$ & $\begin{array}{l}\mathrm{B} \\
(\mathrm{cm})\end{array}$ & $\begin{array}{l}\mathrm{c} \\
(\mathrm{cm})\end{array}$ & $\begin{array}{l}\text { Axial ratio } \\
\text { c/a }\end{array}$ & $\begin{array}{l}\text { Maximum } \\
\text { projection } \\
\text { Sphericity } \\
\left(c^{2} / a b\right)^{1 / 3}\end{array}$ & $\begin{array}{l}\text { Coefficient } \\
\text { Flatness } \\
(\mathrm{c} / \mathrm{a}) \times 100\end{array}$ \\
\hline 1 & 2.9 & 2.2 & 1.2 & 0.4 & 0.6 & 41.4 \\
\hline 2 & 2.1 & 1.8 & 1.4 & 0.7 & 0.8 & 66.7 \\
\hline 3 & 2.2 & 1.6 & 0.9 & 0.4 & 0.6 & 40.9 \\
\hline 4 & 2.0 & 1.8 & 1.0 & 0.5 & 0.7 & 50.0 \\
\hline 5 & 2.6 & 2.3 & 1.1 & 0.4 & 0.2 & 42.3 \\
\hline 6 & 2.2 & 1.8 & 0.8 & 0.4 & 0.5 & 36.4 \\
\hline 7 & 2.0 & 1.4 & 1.3 & 0.7 & 0.8 & 65.0 \\
\hline 8 & 1.9 & 1.5 & 1.3 & 0.7 & 0.6 & 68.4 \\
\hline 9 & 2.1 & 1.4 & 1.1 & 0.5 & 0.7 & 53.4 \\
\hline 10 & 2.5 & 2.0 & 1.5 & 0.6 & 0.8 & 60.0 \\
\hline 11 & 1.8 & 1.3 & 1.1 & 0.6 & 0.1 & 61.0 \\
\hline 12 & 1.8 & 1.7 & 1.2 & 0.7 & 0.8 & 66.6 \\
\hline 13 & 3.0 & 2.3 & 1.2 & 0.4 & 0.6 & 40.0 \\
\hline 14 & 2.3 & 1.5 & 1.2 & 0.5 & 0.7 & 52.2 \\
\hline 15 & 1.8 & 1.6 & 0.8 & 0.4 & 0.6 & 44.4 \\
\hline 16 & 1.7 & 1.4 & 1.0 & 0.6 & 0.7 & 58.8 \\
\hline 17 & 1.6 & 1.4 & 1.0 & 0.6 & 0.8 & 62.5 \\
\hline 18 & 2.5 & 1.7 & 1.5 & 0.6 & 0.8 & 60.0 \\
\hline 19 & 2.3 & 2.0 & 1.2 & 0.6 & 0.7 & 52.2 \\
\hline 20 & 1.5 & 1.2 & 1.1 & 0.7 & 0.9 & 73.3 \\
\hline 21 & 2.0 & 1.5 & 1.2 & 0.6 & 0.9 & 60.0 \\
\hline 22 & 2.0 & 1.2 & 0.8 & 0.4 & 0.5 & 40.0 \\
\hline 23 & 1.9 & 1.5 & 0.8 & 0.4 & 0.6 & 42.1 \\
\hline 24 & 2.0 & 1.5 & 0.7 & 0.4 & 0.5 & 35.0 \\
\hline 25 & 1.9 & 0.8 & 0.6 & 0.3 & 0.6 & 31.6 \\
\hline 26 & 1.7 & 1.2 & 1.0 & 0.6 & 0.8 & 58.8 \\
\hline 27 & 1.6 & 1.1 & 0.7 & 0.4 & 0.3 & 43.8 \\
\hline 28 & 1.4 & 1.2 & 0.5 & 0.4 & 0.5 & 35.7 \\
\hline 29 & 1.7 & 1.4 & 1.1 & 0.67 & 0.8 & 64.7 \\
\hline 30 & 1.9 & 1.6 & 1.2 & 0.6 & 0.8 & 63.2 \\
\hline 31 & 2.0 & 1.5 & 0.7 & 0.4 & 0.5 & 35.0 \\
\hline 32 & 1.8 & 1.3 & 0.8 & 0.4 & 0.6 & 44.4 \\
\hline 33 & 1.5 & 1.4 & 1.0 & 0.77 & 0.7 & 66.7 \\
\hline Mean & & & & & 0.7 & 52.0 \\
\hline
\end{tabular}


Table 5: Summary of Pebble Morphometric Analysis Results at $10^{\circ} 2459.6 \mathrm{~N}$ and $11^{0} 1407.2 \mathrm{E}$

\begin{tabular}{|c|c|c|c|c|c|c|}
\hline S/No & $\begin{array}{l}\text { A } \\
(\mathrm{cm})\end{array}$ & $\begin{array}{l}\text { B } \\
(\mathrm{cm})\end{array}$ & $\begin{array}{l}\mathrm{C} \\
(\mathrm{cm})\end{array}$ & $\begin{array}{l}\text { Axial ratio } \\
\text { c/a }\end{array}$ & $\begin{array}{l}\text { Maximum } \\
\text { projection } \\
\text { sphericity } \\
\left(c^{2} / a b\right)^{1 / 3}\end{array}$ & $\begin{array}{l}\text { Coefficient } \\
\text { Flatness } \\
(\mathrm{c} / \mathrm{a}) \times 100\end{array}$ \\
\hline
\end{tabular}

\begin{tabular}{lllllll}
\hline 1 & 1.9 & 1.5 & 0.9 & 0.5 & 0.7 & 47.4 \\
2 & 2.2 & 1.7 & 1.0 & 0.5 & 0.6 & 45.5 \\
3 & 2.1 & 1.9 & 0.9 & 0.4 & 0.6 & 42.9 \\
4 & 1.7 & 1.5 & 0.8 & 0.5 & 0.6 & 47.1 \\
5 & 2.5 & 2.0 & 1.2 & 0.5 & 0.6 & 48.0 \\
6 & 2.4 & 1.5 & 1.2 & 0.5 & 0.7 & 50.0 \\
7 & 1.6 & 1.2 & 0.7 & 0.4 & 0.6 & 43.8 \\
8 & 2.1 & 1.5 & 1.0 & 0.5 & 0.7 & 47.6 \\
9 & 1.2 & 0.9 & 0.6 & 0.5 & 0.7 & 50.0 \\
10 & 2.7 & 1.2 & 0.9 & 0.3 & 0.6 & 33.3 \\
11 & 2.5 & 1.2 & 0.8 & 0.3 & 0.6 & 32.0 \\
12 & 1.8 & 1.2 & 0.7 & 0.4 & 0.6 & 38.9 \\
13 & 1.7 & 1.1 & 0.4 & 0.3 & 0.4 & 23.5 \\
14 & 1.6 & 1.1 & 0.7 & 0.4 & 0.7 & 43.8 \\
15 & 1.7 & 1.0 & 0.6 & 0.4 & 0.6 & 35.3 \\
16 & 1.8 & 1.4 & 1.0 & 0.6 & 0.7 & 55.6 \\
17 & 2.6 & 1.9 & 1.0 & 0.4 & 0.6 & 38.5 \\
18 & 1.8 & 1.3 & 0.8 & 0.4 & 0.6 & 44.4 \\
19 & 2.1 & 1.2 & 0.9 & 0.4 & 0.7 & 42.9 \\
20 & 2.1 & 1.2 & 1.0 & 0.4 & 0.7 & 47.6 \\
21 & 2.0 & 1.3 & 1.1 & 0.6 & 0.7 & 55.0 \\
22 & 1.7 & 1.2 & 1.0 & 0.6 & 0.8 & 58.8 \\
23 & 2.0 & 0.9 & 0.5 & 0.3 & 0.5 & 25.0 \\
24 & 1.8 & 1.3 & 0.6 & 0.3 & 0.5 & 33.3 \\
25 & 2.2 & 1.6 & 1.1 & 0.5 & 0.3 & 50.0 \\
26 & 1.9 & 1.4 & 0.7 & 0.4 & 0.5 & 39.8 \\
27 & 1.4 & 1.0 & 0.8 & 0.6 & 0.8 & 57.1 \\
28 & 1.6 & 0.9 & 0.6 & 0.4 & 0.6 & 37.5 \\
29 & 1.7 & 1.1 & 0.5 & 0.3 & 0.5 & 29.4 \\
30 & 1.8 & 1.5 & 0.8 & 0.4 & 0.6 & 44.4 \\
31 & 1.8 & 1.3 & 1.0 & 0.6 & 0.8 & 55.6 \\
32 & 2.0 & 1.2 & 0.7 & 0.4 & 0.6 & 35.0 \\
33 & 2.6 & 1.7 & 0.8 & 0.3 & 0.5 & 30.8 \\
Mean & & & & & 0.6 & 42.7 \\
\hline & & & & & & \\
\hline
\end{tabular}


Table 6: Summary of Pebble Morphometric Analysis Results $10^{\circ} 2353.8 \quad \mathrm{~N}$ and $11^{0} 1452.1 \mathrm{E}$

\begin{tabular}{|c|c|c|c|c|c|c|}
\hline S/No & $\begin{array}{l}\mathrm{a} \\
(\mathrm{cm})\end{array}$ & $\begin{array}{l}\mathrm{B} \\
(\mathrm{cm})\end{array}$ & $\begin{array}{l}\mathrm{c} \\
(\mathrm{cm})\end{array}$ & $\begin{array}{l}\text { Axial } \\
\text { ratio } \\
c / a\end{array}$ & $\begin{array}{l}\text { Maximum } \\
\text { projection } \\
\text { Sphericity } \\
\left(c^{2} / a b\right)^{1 / 3}\end{array}$ & $\begin{array}{l}\text { Coefficient } \\
\text { Flatness }(c / a) \\
\text { x100 }\end{array}$ \\
\hline 1 & 3.2 & 2.5 & 1.2 & 0.4 & 0.6 & 37.5 \\
\hline 2 & 2.2 & 1.8 & 0.9 & 0.4 & 0.6 & 40.9 \\
\hline 3 & 1.7 & 1.5 & 1.0 & 0.6 & 0.7 & 58.8 \\
\hline 4 & 2.1 & 1.6 & 0.9 & 0.4 & 0.6 & 42.8 \\
\hline 5 & 1.5 & 1.2 & 0.6 & 0.4 & 0.6 & 40.0 \\
\hline 6 & 1.8 & 1.5 & 1.2 & 0.7 & 0.8 & 66.7 \\
\hline 7 & 2.0 & 1.4 & 0.7 & 0.3 & 0.6 & 35.0 \\
\hline 8 & 1.7 & 1.3 & 0.7 & 0.4 & 0.6 & 41.2 \\
\hline 9 & 2.1 & 1.5 & 1.0 & 0.5 & 0.7 & 47.6 \\
\hline 10 & 1.7 & 1.2 & 1.0 & 0.6 & 0.8 & 58.8 \\
\hline 11 & 1.8 & 1.4 & 0.8 & 0.4 & 0.6 & 44.4 \\
\hline 12 & 1.8 & 0.9 & 0.6 & 0.3 & 0.6 & 33.3 \\
\hline 13 & 1.6 & 1.2 & 1.0 & 0.6 & 0.8 & 62.5 \\
\hline 14 & 1.8 & 0.9 & 0.6 & 0.3 & 0.6 & 33.4 \\
\hline 15 & 1.6 & 1.0 & 0.8 & 0.5 & 0.7 & 50.0 \\
\hline 16 & 2.1 & 0.9 & 0.6 & 0.3 & 0.4 & 28.5 \\
\hline 17 & 2.4 & 0.7 & 0.5 & 0.2 & 0.5 & 20.8 \\
\hline 18 & 2.0 & 1.6 & 1.2 & 0.6 & 0.7 & 60.0 \\
\hline 19 & 1.6 & 1.2 & 0.9 & 0.6 & 0.6 & 56.3 \\
\hline 20 & 2.3 & 1.4 & 0.8 & 0.4 & 0.7 & 34.8 \\
\hline 21 & 1.6 & 1.4 & 0.9 & 0.6 & 0.8 & 56.3 \\
\hline 22 & 1.6 & 1.2 & 1.0 & 0.6 & 0.6 & 62.5 \\
\hline 23 & 2.0 & 1.5 & 0.9 & 0.5 & 0.9 & 45.0 \\
\hline 24 & 1.8 & 1.1 & 0.8 & 0.4 & 0.6 & 44.4 \\
\hline 25 & 2.1 & 1.6 & 0.9 & 0.4 & 0.7 & 42.8 \\
\hline 26 & 2.0 & 1.5 & 1.0 & 0.5 & 0.5 & 50.0 \\
\hline 27 & 1.7 & 1.5 & 0.6 & 0.5 & 0.6 & 35.3 \\
\hline 28 & 2.0 & 1.2 & 0.7 & 0.5 & 0.7 & 35.0 \\
\hline 29 & 1.7 & 1.1 & 1.0 & 0.6 & 0.8 & 58.8 \\
\hline 30 & 1.5 & 1.0 & 0.9 & 0.6 & 0.7 & 60.0 \\
\hline 31 & 2.2 & 1.5 & 0.8 & 0.4 & 0.6 & 36.4 \\
\hline 32 & 1.8 & 1.3 & 0.7 & 0.4 & 0.7 & 38.9 \\
\hline 33 & 1.9 & 1.2 & 1.0 & 0.9 & 0.8 & 52.6 \\
\hline Mean & & & & & 0.7 & 45.8 \\
\hline
\end{tabular}

\section{DISCUSSION}

\section{Univariate grain size parameters}

The graphic mean size for the various sample of Gombe Formation range from $0.8 \phi$ to $3.1 \phi$, which indicate coarse to very fine grained. Freidman (1967) pointed out that grained size distributions are controlled by processes rather than environment. Therefore the fluctuations in mean values suggest change in energy of the depositional conditions. Sorting is important factor that is use for environmental analysis. It is very significant in differentiating between fluvial and wave deposits (Freidman 1967). Sorting for various samples of the Gombe Formation range from $0.37 \phi$ to $1.01 \phi$, this indicates well to poorly sorting. However moderately to well sorted values predominate. The well to moderately sorted nature of Gombe Formation may suggest 
reworking by wave activities, which removed clay material from the sand (Mason and Folk 1958; Freidman 1967).

The values of skewness for various samples range from $0.31 \phi$ to $0.4 \phi$ which indicates negative skewed to positive skewed. However the positive skewed values predominate. The predominance of positive skewed in coarsening upward deposit of Gombe Formation suggest transitional environment. This interpretation is supported by the work of Freidman (1967 and1979), in which he suggested positive skewed for deeper part of the continental shelf/lagoon and beach where abundance of fine exceeds the energy available for dispersing them. The values for kurtosis from various sample range from $0.56 \phi$ to $2.05 \phi$ (very platykurtic to very leptokurtic) with an average of $1.3 \phi$ (leptokurtic). Kurtosis has little Geologic information that could be obtained (Boggs 2006), however, the fluctuation in values may suggest change in energy of depositional medium ( Abdel-Wahab 1988, Shetima et al., 2012).

For the discrimination between aeolian and littoral (intertidal zone) environments, the Y1equation is used. Sahu (1964) suggest that when $Y 1$ is less than 2.7411 it is an Aeolian deposit whereas if $\mathrm{Y} 1$ is greater than - 2.7411 a beach environment is suggested. A Aeolian environment was infer, since $62.5 \%$ of the values of $\mathrm{Y} 1$ are greater than -2.7411 (Table 3). This may suggest aeolian deposits associated with bar deposit. For the discrimination between beach (backshore) and shallow agitated marine environments (sub-tidal environment) the $\mathrm{Y} 2$ equation is used. If the value of $Y 2$ is less than 65.3650 a beach deposition is suggested, whereas if it is greater than 65.3650, a shallow agitated marine environment is inferred. Shallow agitated marine environment is suggested since $93.25 \%$ values of $\mathrm{Y} 2$ calculated are greater than 65.3650 (Table 3). This agitated marine environment may be taken to be a deltaic realm, where Gombe Formation is inferred as fluvial-deltaic in nature. For discrimination between shallow marine and the fluvial environments, the discrimination equation of $\mathrm{Y} 3$ is used. If $Y 3$ is less than -7.419 the sample is identified as a fluvial deposits whereas if $\mathrm{Y} 3$ is greater than -7.419 the sample is identified as a shallow marine deposit. The analyzed results showed $93.75 \%$ of the plotted $\mathrm{Y} 3$ values from the total number of samples has values more than -7.419, suggestive of shallow marine environment while $6.25 \%$ has $\mathrm{Y} 3$ less than -7.419 inferring fluvial setting (Table 3 ).
The result obtained from $\mathrm{Y} 2, \mathrm{Y} 3$ and probability plot have supported the earlier interpretation of the positive skewed values as transitional environment rather than fluvial environment.

\section{Bivariate grain size parameters}

The bivariate plots of standard deviation vs. skewness is based on Friedman (1979) which distinguishes Inland Dune Sand from Beach Sand, the plots indicates that $68.75 \%$ of the samples plotted within the river field and $31.25 \%$ of the samples plotted within the Beach Sand field (Fig. 3). The dominance of samples within Inland Dune field was due to river pumps of tremendous load of fines into the nearshore environment and the available energy was unable to remove them (Friedman, 1967)

Plotting of the three discriminate functions ( $Y 1$, Y2 and Y3) as bivariate scatter plots was used to improve the success rate and refinement of the discrimination of the depositional environment. A bivariate plot of $\mathrm{Y} 1$ and $\mathrm{Y} 2$ (Fig. 5) show most samples to be littoral or shallow agitated marine environment. A bivariate plot of Y2 and Y3 (Fig.6) show that most samples are of fluvial/deltaic environment.

\section{Probability plots}

According to Visher (1969) different sand population are of environmental value. The sand population curves are characteristic of either fluvial, beach or wave zone. Two sand populations are characteristic of fluvial setting, while three sand populations are characteristic of shallow marine setting. Probability plots of samples from bedded facies of Gombe Formation (Figs. 4A, B and C) show three sand populations which indicate deposit of shallow marine environment. Samples of red sandstone facie show two sand populations which suggest fluvial environment (Fig.4D).

Fourteen samples obtained from coarsening upward units have three sand populations. Eleven of the samples have saltation population from 2.0 to 3.5 and little suspension population (Fig. 4A and B) similar to Visher (1969) figure 9A. This type of curves were interpreted as marine sand from wave zone, while sample 12 and 14 have saltation population between 2.7 to 3.9 and 10 percent surface creep (Fig.4C) similar to Visher (1969) figure 13A which was interpreted as offshore marine sand in the area of tidal delta at depth of 3.05 to $12.2 \mathrm{~m}$. 


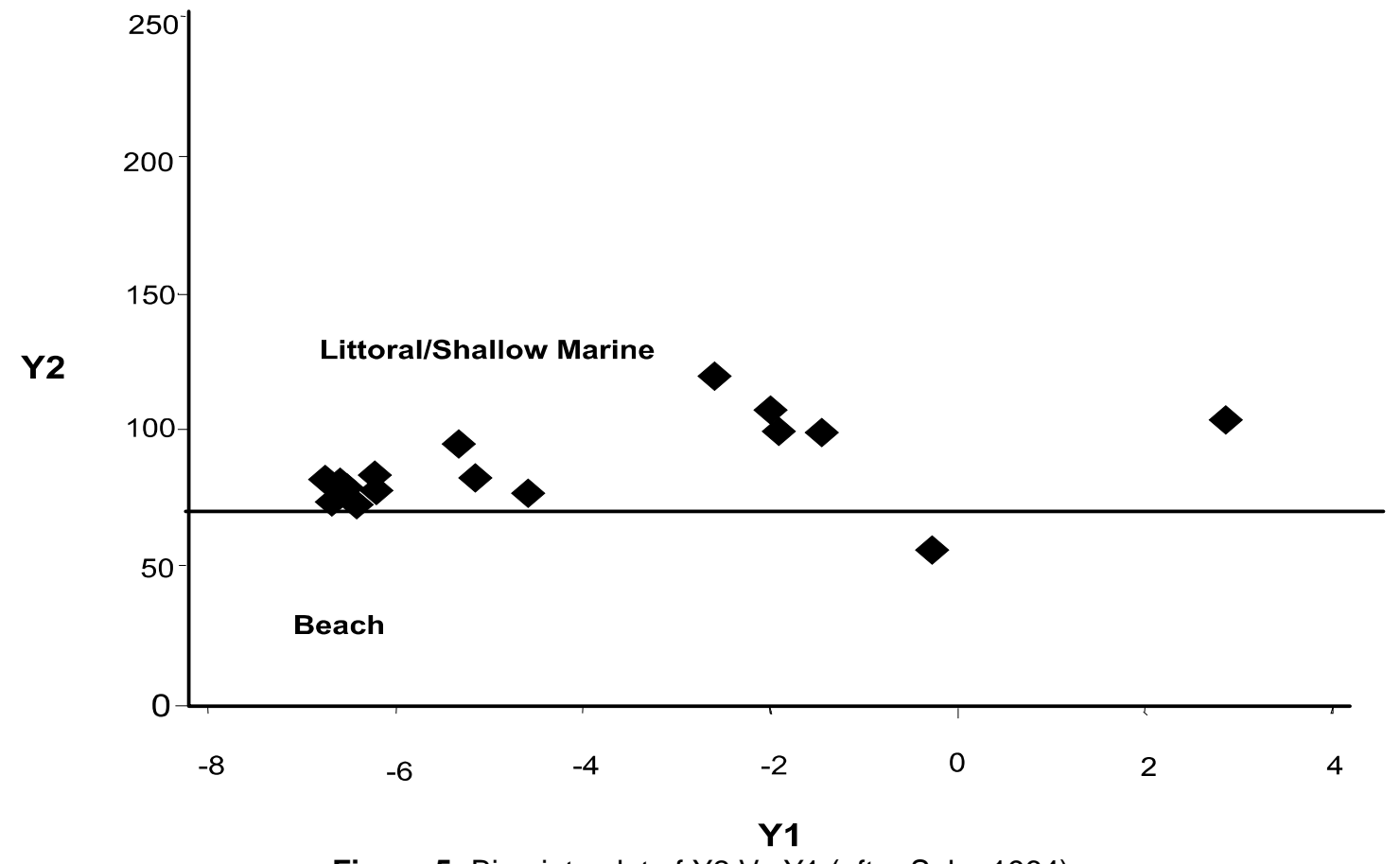

Figure 5: Bivariate plot of Y2 Vs Y1 (after Sahu 1964)

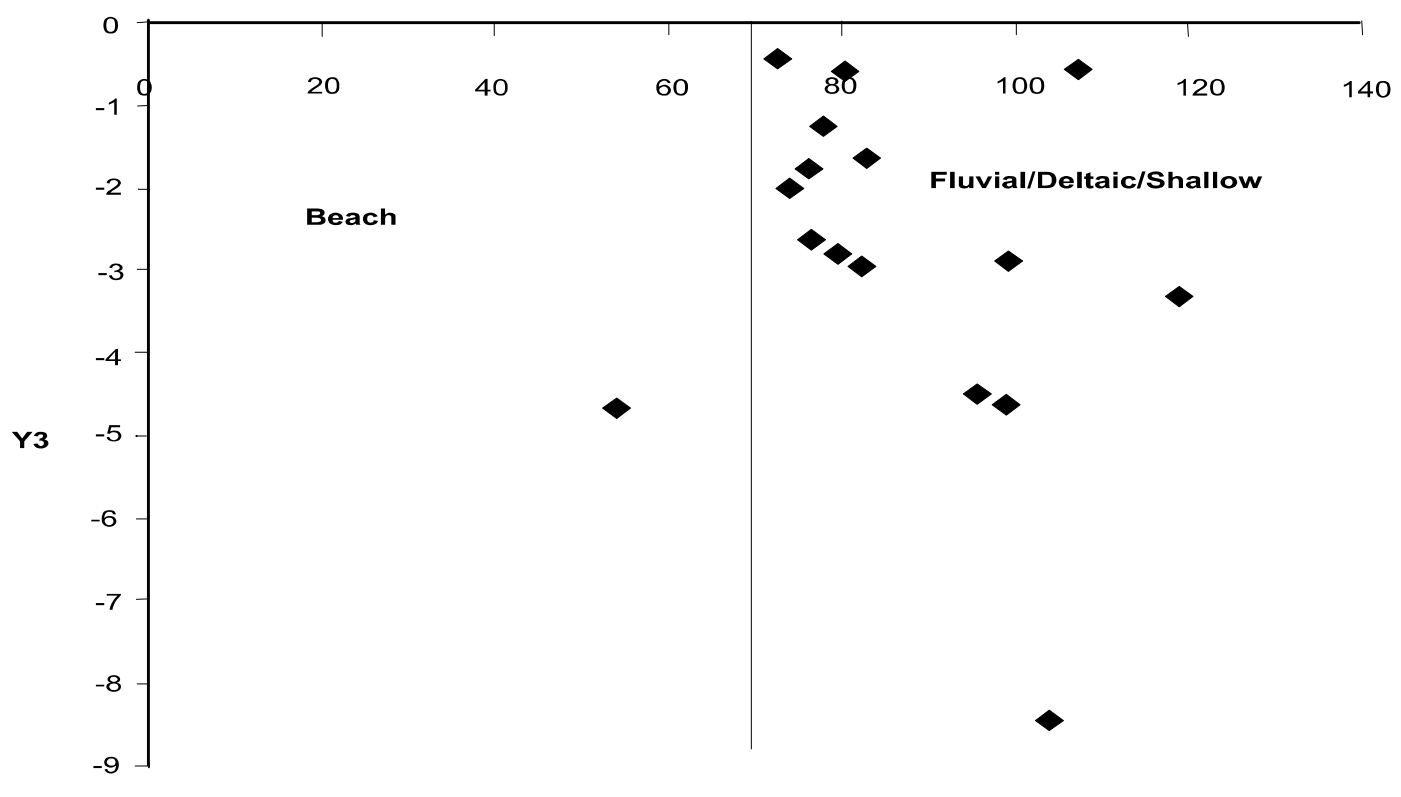

Y2

Figure 6: Bivariate plot of Y3 Vs Y2 (after Sahu 1964)

\section{Pebbles morphometric}

Maximum projection sphericity and coefficient of flatness are environmentally diagnostic (Stratten 1974). Pebbles from Gombe Formation were measured and the value of maximum projection sphericity and coefficient of flatness were calculated to determine its depositional environment. The pebbles have mean maximum projection sphericity of $0.646,0.627$ and 0.667 table 4,5 and 6 respectively and mean coefficient of flatness of 52.013. 42.714 and 45.796 table 4.5 and 6 respectivelv.
Sphericity is a measure of the approach of a pebble to sphere. It can be measured in different ways (Krumbein, 1941, Sneed and Folk 1958). In this study the maximum projection sphericity of Sneed and Folk has been used. The maximum projection sphericity values for range from 0.4 to 0.9 with mean value of 0.65 . The average value of maximum projection sphericity is exactly at a line of separation between beach and fluviatile of Stratten (1974), indicating beach environment. 
The Coefficient Flatness values range from 20.8 to 73 with mean value of 46.8 . The average value of coefficient flatness is slightly above the 45 coefficient flatness line of Stratten (1974), indicating fluvial environment.

Each individual pebble sample studied here was plotted on a Sneed and Folk (1958) (Fig. 7) diagram using the "TRI - PLOT" programme (Graham and Midgley, 2000) and the average percentages of the form classes were calculated and shown in Table 7 . The study of form indices has shown the dominance of the following form classes Bladed, Compact - Bladed, Compact - Elongated and Platy. According to Dobkins and Folk (1970) Compact, Compact - Bladed and Compact - Elongated forms are indicative of fluvial action. Blades are common on both beach and fluvial environment. The study of the form indices has shown that pebbles of the Gombe Formation were shaped in a fluvial environment.

Table 7: Percentages of pebbles forms class categories

\begin{tabular}{|l|l|l|}
\cline { 2 - 3 } \multicolumn{1}{c|}{} & Count & Percent (\%) \\
\hline Compact & 1 & 1.0 \\
\hline Compact-Platy & 5 & 5.1 \\
\hline Compact-Bladed & 16 & 16.2 \\
\hline Compact-Elongate & 12 & 12.1 \\
\hline Platy & 11 & 11.1 \\
\hline Bladed & 38 & 38.4 \\
\hline Elongate & 11 & 11.1 \\
\hline Very-Platy & 0 & 0.0 \\
\hline Very-Bladed & 2 & 2.0 \\
\hline Very-Elongate & 3 & 3.0 \\
\hline
\end{tabular}



$(\mathrm{a}-\mathrm{b}) /(\mathrm{a}-\mathrm{c})$

Figure 7: Sneed and Folk (1958) diagram showing shape based on the parameters $C=$ Compact, $C P=C o m p a c t$ Platy, $\mathrm{CB}=$ Compact - Bladed, $\mathrm{CE}=$ Compact - Elongate, $\mathrm{P}=$ Platy, $\mathrm{B}=$ Bladed, $\mathrm{E}=$ Elongate, $\mathrm{VP}=$ Very Platy, $\mathrm{VB}=$ Very Bladed, $\mathrm{VE}=$ Very Elongate.

\section{CONCLUSION}

The numerical values used in an analysis of all studied granulometric parameters, pebble morphometric parameters and bivariate plots as well as sand population all point to a series of related subenvironment in transitional environment with subordinate results of fluvial setting. The various characteristic of the sediment as fine arain. verv fine arain Dositive skewness and well sorted nature of Gombe Formation are in agreement with early studied by Shetima et al. (2012)

\section{REFERENCES}

Abdel-Wahab, A., 1988. Lithofacies and diagenesis of the Nubia Formation at Central Eastern desert, Egypt (abstract) $9^{\text {th }}$ EGP. Expl. Conf. Cairo. 2023 Nov. IIA 9. 
Abubakar, M. B., 2006. Biostratigraphy, palaeoenvironment and organic geochemistry of the Cretaceous sequences of the Gongola Basin, Upper Benue Trough, Nigeria. Unpublished Ph.D. Thesis, Abubakar Tafawa Balewa University, Bauchi, 139-140.

Benkhelil, J., 1989. The origin and evaluation of the cretaceous Benue Trough (Nigeria). Journal African Earth Sciences, 8, 251-282.

Boggs, S. Jr., 2006. Principles of Sedimenology and Stratigraphy: Pearson Education Inc. 31.

Carter, J. D., Barber, W., Tait, E. A. and Jones, G. P., 1963. The geology of parts of Adamawa, Bauchi and Borno province in North-Eastern Nigeria. Bulletin Geological Survey, Nigeria, 30, 1-99.

Cratchley, G. R., Louis, P and Ajakaiye, D. E., 1984. Geophysical and geological evidence for the Benue-Chad Basin Cretaceous rift valley system and its tectonic implication. Journal of African Earth Science, 2, 141-150.

Dobkins, J. E and Folk, R. L., 1970. Shape development on Tahiti-nui. Journal of Sedimentary Petrology, 40, 1167-1203.

Fitton, J. G., 1983. Active versus passive continental rifting evidence from the West African Rift System. In Morgan, P. And Baker,B.H.,(eds) Processes of Continental Rifting. Tectonophysics, 94, 473-481.

Folk, R. L., 1974. Petrology of Sedimentary Rocks. Hemphill Publishing Company, Austin Texas.

Folk, R. L and Ward, W. C., 1957. Brazos River bar, a study in significance to grain size parameters. Journal of Sedimentary petrology, 30, 514-529.

Friedman, G. M., 1967. Dynamic processes and statistical parameters compared for size frequency distribution of beach and river sands. Journal of Sedimentary Petrology, 37, 327-354.

Friedman, G. M., 1979. Differences in size distributions of populations of particles among sands of various origins. Sedimentology, 26, 3-32.

Ganik, G. J., 1992. Regional framework, structural and petroleum aspects of rift basins in Niger, Chad and Central African Republic (CAR). Tectonophysics, 213, 169-189.

Graham, D. J and Midgley, N. G., 2000. Graphical representation of Particle shape using Triangular diagrams: and Excel spreadsheet method. Earth Surface Processes and Landforms, 25, 1473 - 1477.

Grant, N. K., 1971. South Atlantic, Benue Trough and Gulf of Guinea Cretaceous Triple Junction. Bulletin of Geological Society of America, 82, 2295-2298.
Guiraud, R and Maurin, J. C., 1992. Early Cretaceous rifts of westerns and central Africa an Overview. Tectonophysics, 282, 39-82.

Hamidu, I., 2012. The Campanian To Maastrichtian Stratigraphic Succession In The Cretacious Gongola Basin Of North-Eastern Nigeria. Unpublished Ph.D. Thesis, Ahmadu Bello University, Zaria, 41-177.

King, L. C., 1950. Outline and disruption of Gondwanaland. Geological Magazine 87, 353362.

Kogbe, C. A., 1976. Paleogeographic history of Nigeria from Albian times. In: Kogbe CA (ed), Geology of Nigeria. Elizabethan Publishers, Lagos, 1535.

Krumbein, W. C., 1941. Measurement and geologic significance of shape and roundness of sedimentary particles Journal of sedimentary petrology, 11, 64-72.

Mason, C. C and Folk, R. L., 1958. Differentiation of beach dune and eolian flat environments by size analysis, Mustand Island, Texas: jour. Sed. Petrology, 28, 211-226.

Nwajide, C. S., 2013. Geology of Nigeria's Sedimentary Basins. CSS Bookshops Ltd, Lagos, Nigeria, 565.

Offodile, M. E., 1976. The geology of the Middle Benue, Nigeria. Palaeontological Institute, University Uppsala, Special Publication 4: 1-166.

Olade, M. A., 1975. Evolution of Nigeria's Benue Trough: a tectonic model. Geological Magazine, $112,575-583$.

Popoff, M., Wiedmann, J and De Klasz, I., 1986. The Upper Cretaceous Gongila and Pindiga Formations, northern Nigeria: subdivision, age, stratigraphic correlations and paleogeographic implications. Ecologae Geologicae Helvetiae, 79, 343-363.

Sahu, B. K., 1964. Depositional Mechanism for the size analysis of clastic sediments. Journal Sedimentary Petrology, 34, 73-83.

Sneed, E. D and Folk, R. L., 1958. Pebbles in the lower Colarado River, Texas: a study in particle morphogenesis: J. Geol., 66, 144-150.

Stratten, T., 1974. Notes on the application of shape parameters to differentiate between Beach and River deposits. Southern African Trans, Geological Society South African 77. 59-64.

Tukur, A., Samaila, N. K., Grimes, S. T., Kariya, I. I and Chaanda, M. S., 2015. Two member subdivision of the Bima Sandstone, Upper Benue Trough: Based on sedimentological data. Journal of African Earth Sciences, 104, 140-158. 
Visher, G. S., 1969. Grain size distributions and depositional processes. Journal of Sedimentary Petrology, 39, 1074-1106.

Wentworth, C. K., 1922. A scale of grade and class terms for clastic sediments. Journal Geology, 30, 377-392.

Zaborski, P. M., 1997. Guide to the Cretaceous System in the Upper Part of the Upper Benue Trough, North-eastern Nigeria. African Geosciences Review 10, (1\&2): 1322.

Zaborski, P. M., Ugodulunwa, F., Idornigie, A., Nnabo, P and Ibe, K., 1997. Stratigraphy and structure of the Cretaceous Gongola Basin, North-eastern
Nigeria. Bulletin of Centre for Researches Elf Exploration and Production, 21, (1): 153-185.

Zaborski, P. M., 1998. A review of the Cretaceous system in Nigeria. African Geosciences Review.

Zingg, T., 1935. Beitrage Zur Schotteranalyse: Schweiser Mineralog U. Petrog. Mitt. Bd. 15: 38110. 